\title{
Modeling and Simulation of High-bandwidth Si-based MOS/SOI Photodetectors
}

\author{
C.-Y. Liang", B.-C. Hsu' , C.-H. Lin', S. T. Chang ${ }^{c}$, and C. W. Liu \\ ${ }^{a}$ Graduate Institute of Electro-Optical Engineering, National Taiwan University, \\ Taipei, Taiwan, R. O. C. \\ ${ }^{6}$ Department of Electrical Engineering and Graduate Institute of Electronics Engineering, \\ National Taiwan University, Taipei, Taiwan, R. O. C. \\ ${ }^{c}$ Department of Electronic Engineering, Chung Yuan Christian University, \\ Chung-Li, Taiwan, R. O. C. \\ Tel: 886-2-23635251 ext. 515/ Fax: 886-2-23638247 / E-mail: chee@cc.ee.ntu.edu.tw
}

The bandwidth of bulk-MOS photodetectors (PD) can not reach GHz due to the absorption length of $850 \mathrm{~nm}$ lightwave in $\mathrm{Si}(\sim 22 \mathrm{um})$ is much larger than the Si depletion width even when the device is deeply depleted [1][2]. Therefore, most photo-generated electron-hole pairs are located in the bulk neutral region and are collected by gate electrode through the slow diffusion process. The large diffusion current seriously limits the device bandwidth and the applications. In this work, a novel MOS PD structure is proposed to eliminate the diffusion current by taking advantage of SOI technology (Fig. 1). The thick buried oxide stops the diffusion current from substrate and the thin absorption layer makes sure that the device is fully-depleted during operation. The absorption region and ground electrode are separated by oxide and connected by buffer layer. Note that the grid structure of $\mathrm{Al}$ gate electrode allows the light directly exposures on the absorption region. The device simulation is carried out by commercial software ISE [3].

Since the diffusion current is eliminated in SOI-MOS PD to increase device speed and bandwidth, total photocurrent is also reduced (Fig. 2). Thicker absorption region will increase the responsivity but reduces bandwidth if the absorption layer is thicker than the depletion width. Since the tunneling MOS diode is deep depleted under inversion bias (inset of Fig. 2), lighter doping in absorption region and higher operation voltage $\left(\mathrm{V}_{8}\right)$ will increase the depletion width. Fig. 3 is the band diagram of SOI-MOS PDs along hole current path from oxide/Si interface to ground contact for devices with different buffer layer doping concentration $\left(10^{16}, 10^{18}\right.$, and $10^{20}$ $\mathrm{cm}^{-3}$ ). Electron-hole pairs are generated in absorption layer and the holes are swept into buffer layer (flat band region) and finally collected by ground. The device with $10^{16} \mathrm{~cm}^{-3}$ doping has a energy barrier because the depletion width extends into the lightly doped buffer layer. Therefore, the photo-generated carriers in the middle of the device are blocked and couldn't transport into ground contact. The transient analysis is also carried out and shows the same results (Fig. 4). The photocurrent of device with $10^{20} \mathrm{~cm}^{-3}$ buffer layer doping rises and falls quickest under 5 ps optical impulse due to the smallest resistivity and RC delay time. Note that when devices are operated in high speed, the SOI-MOS PD generates more photocurrent than the bulk device, unlike the result in DC condition (Fig. 2). The inset of Fig. 4 shows the experimental result of a typical bulk MOS PD with 2 ns FWHM.

The frequency response model of SOI-MOS PDs is also derived. By considering the grid structure of Al gate electrode and the device symmetry, photo-generated carriers are divided into three regions (Fig. 5). Carriers in the three regions have the same transport time within absorption region but different depletion capacitance and series resistance in buffer layer. The total frequency response is obtained by linearly adding them together, as shown in Fig. 6 [4]. The analytical model precisely fits the computer simulation results (Fig. 7). The bandwidth of the MOS/SOI PD is up to $22 \mathrm{GHz}$. The parameter values used in the analytical model are given in Table. 1. For device with $10^{16}$ buffer layer doping, the efficiency is only $1 / 5$ as compared to other devices because the ground contact only collects the hole generated in the outer part, where the area is $1 / 5$ of the total active area. In DC condition, however, the carriers generated in the middle part can transport to ground contact, so the DC photocurrent are the same for all devices (inset of Fig. 7.).

In conclusion, the novel SOI-MOS PDs can have high bandwidth $(22 \mathrm{GHz}$ ) and are fully compatible with ULSI technology. The device structure could be optimized by tuning the doping and thickness of epi-layers. The absorption layer can be $\mathrm{Si}, \mathrm{SiGe}$ and quantum structures to extend the device detection wavelength to $1.3,1.5 \mu \mathrm{m}$ and even for $10 \mu \mathrm{m}$ infrared detection.

[1] B.C. Hsu et al., IEEE Electron Device Letters, vol. 24, pp. 318-320, 2003.

[2] B.-C. Hsu et al., IEDM Tech. Dig., pp.91-94, 2002.

[3] ISE TCAD 8.0. [4] Weishu Wu et al., J. of Lightwave Tech., vol. 14, pp. 2778-2785, 1996. 


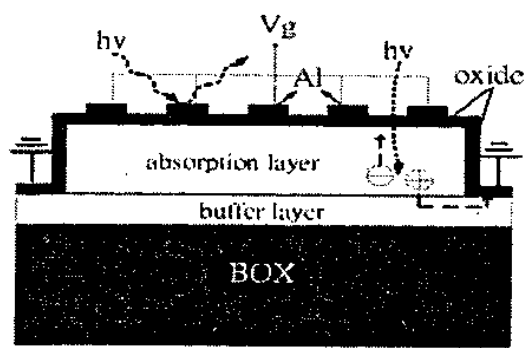

Fig. 1 The proposed SOI-MOS PD structure. The absorption bayer is fully-depleted during operation Note that there is no beam generation and photo current under Al contact because of reflection.

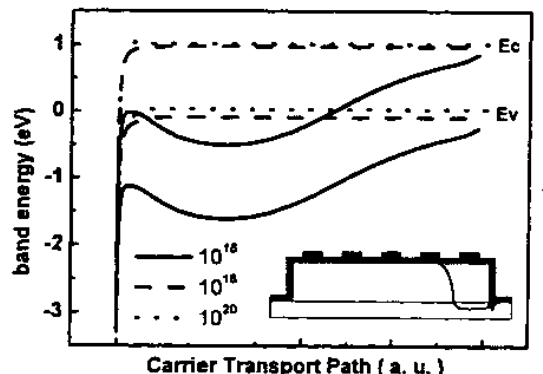

Fig. 3 Band diagrams of the SOI-MOS PDs with different buffer layer doping. The inset shows the route of hole current. A energy barrier for hole is observed for device with $10^{16}$ buffer layer doping.

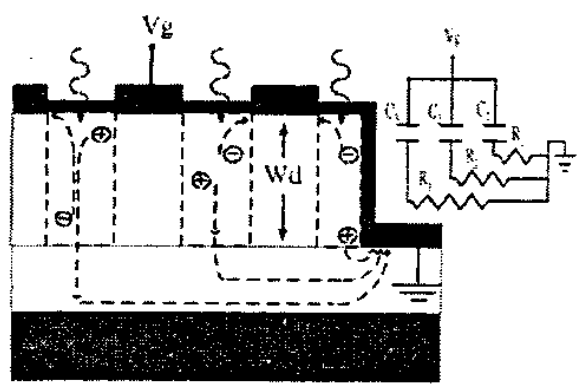

Fig. 5 The right half side of SOI PD is divided by $\mathrm{Al}$ contacts into three regions. Current in each region has its own depletion capacitance, series resistance and frequency response.

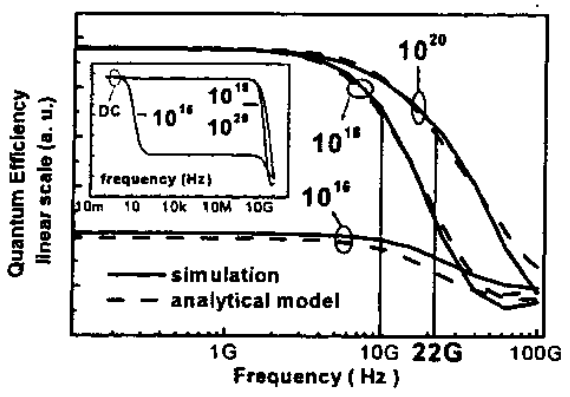

Fig. 7 Frequency responses of devices with different buffer layer doping concentration. The device with higher doping concentration yields larger bandwidth. The efficiency of doping $10^{16}$ device drops near DC because the buffer layer is also depleted and most holes are blocked by the energy barrier

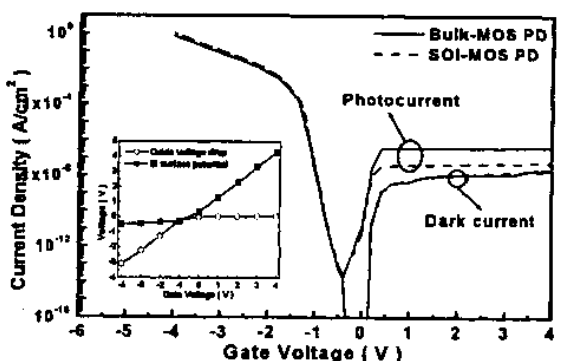

Fig. 2 The dark and photo current of bulk and SOI devices under $850 \mathrm{~nm}$ light exposure. The inset shows that under inversion bias $\left(\mathrm{V}_{\mathrm{z}}>0\right)$, most voltage drops falls on $\mathrm{Si}$ substrate and the depletion width increases with $\mathrm{V}_{\mathrm{z}}$

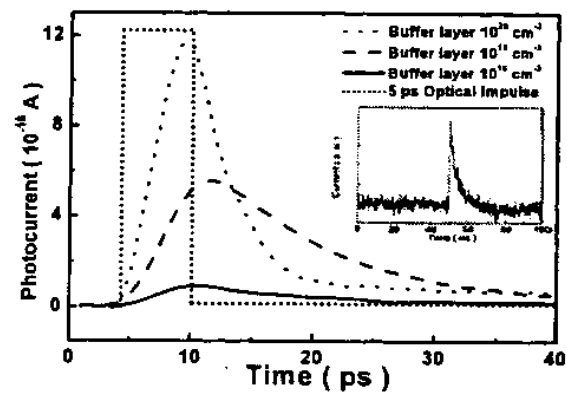

Fig. 4 Transient analysis of SOI devices with 5 ps optical impulse. The device with highest buffer layer doping has shortest falling time and largest photocurrent. The inset shows that the bulk device has a 2 ns FWHM measured which is much lager than the plot scale.

$$
\begin{aligned}
& J(\omega)=\sum_{i=1}^{3} \frac{q}{W_{d}}\left[\frac{v_{n} N(\omega)+v_{p} P(\omega)}{1+j \omega C_{j} R_{\mathrm{i}}}\right] \\
& N(\omega)=\frac{\eta \mathrm{P}}{h v}\left[\frac{e^{-\alpha W_{0}}\left(e^{-i \omega^{\omega}}-1\right)}{j \omega}+\frac{\left(1-e^{-i \omega \frac{W_{-}}{v_{-}}-\alpha V_{2}}\right)}{j \omega+\alpha \nu_{n}}\right]
\end{aligned}
$$

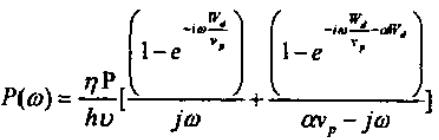

Fig. 6 The proposed analytical solutions for MOS/SOI Photodetectors. $C$ and $R$ are functions of width of $A$

\begin{tabular}{|c|c|c|}
\hline W. & absorption thickriess & $5 \times 10^{-5} \mathrm{~cm}$ \\
\hline$v_{u}$ & electron saturation velocity & $1 \times 10^{7} \mathrm{~cm} / \mathrm{s}$ \\
\hline$P_{r}$ & hole saturation velocky & $6 \times 10^{6} \mathrm{~cm} / \mathrm{s}$ \\
\hline $2 c_{1}=c_{2}=c_{1}$ & capacitance $\equiv A \frac{\varepsilon_{\varepsilon}}{W_{\alpha}}$ & $0.2 \mathrm{fF}$ \\
\hline $\begin{array}{l}R_{1} \\
R_{3} \\
R_{2}\end{array}$ & $\begin{array}{l}\begin{array}{l}\text { resistance for } 1 \mathrm{E} 20 \\
\text { doping } \\
=\end{array} \frac{1}{A} \rho \\
\end{array}$ & $\begin{array}{ll}300 & \\
2 k & \Omega \\
40 k\end{array}$ \\
\hline$\alpha$ & absorption coefticient & $382 \mathrm{~cm}^{\prime \prime}$ \\
\hline$\eta$ & quantum efrieiency & 0.024 \\
\hline $\mathbf{P}$ & light intensity & $1 \times 10^{-6} \mathrm{~W} / \mathrm{cm}^{2}$ \\
\hline he & light energy & $\begin{array}{l}1.450 \mathrm{eV} \\
\text { For } 850 \mathrm{~nm}\end{array}$ \\
\hline
\end{tabular}
contact and space between $\mathrm{Al}$ contacts.

Table 1. Parameter values used in analytical solution. The resistance values are only for the device with $10^{20}$ buffer layer doping 